\title{
Dynamic control allocation using constrained quadratic programming
}

\author{
Ola Härkegård \\ Control \& Communication \\ Department of Electrical Engineering \\ Linköpings universitet, SE-581 83 Linköping, Sweden \\ WWW: http://www. control.isy.liu.se \\ E-mail: ola@isy.liu.se
}

16th February 2004

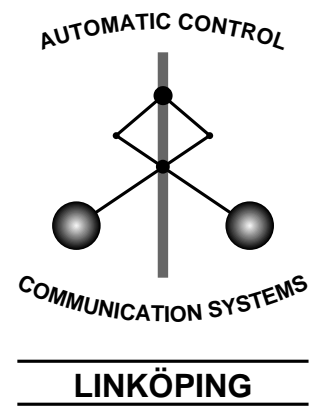

Report no.: LiTH-ISY-R-2594

Submitted to AIAA Guidance, Navigation, and Control

Conference, 2002

Technical reports from the Control \& Communication group in Linköping are available at http://www. control.isy.liu.se/publications. 


\begin{abstract}
Control allocation deals with the problem of distributing a given control demand among an available set of actuators. Most existing methods are static in the sense that the resulting control distribution depends only on the current control demand. In this paper we propose a method for dynamic control allocation, in which the resulting control distribution also depends on the distribution in the previous sampling instant. The method extends the traditional generalized inverse method by also penalizing the individual actuator rates. Its main feature is that it allows for different control distributions during the transient phase of a maneuver and during trimmed flight. The control allocation problem is posed as a constrained quadratic programming problem which provides automatic redistribution of the control effort when one actuator saturates in position or in rate. When no saturations occur, the resulting control distribution coincides with the control demand fed through a linear filter which can be assigned different frequency characteristics for different actuators.
\end{abstract}

Keywords: control allocation, aircraft control, constrained control 


\title{
DYNAMIC CONTROL ALLOCATION USING CONSTRAINED QUADRATIC PROGRAMMING
}

\author{
Ola Härkegård, member AIAA \\ Div. of Automatic Control, Linköpings universitet, Sweden
}

\begin{abstract}
Control allocation deals with the problem of distributing a given control demand among an available set of actuators. Most existing methods are static in the sense that the resulting control distribution depends only on the current control demand. In this paper we propose a method for dynamic control allocation, in which the resulting control distribution also depends on the distribution in the previous sampling instant. The method extends the traditional generalized inverse method by also penalizing the individual actuator rates. Its main feature is that it allows for different control distributions during the transient phase of a maneuver and during trimmed flight. The control allocation problem is posed as a constrained quadratic programming problem which provides automatic redistribution of the control effort when one actuator saturates in position or in rate. When no saturations occur, the resulting control distribution coincides with the control demand fed through a linear filter which can be assigned different frequency characteristics for different actuators.
\end{abstract}

\section{Introduction}

In recent years, nonlinear flight control design methods, like dynamic inversion ${ }^{1}$ and backstepping, ${ }^{2}$ have gained increased attention. These methods result in control laws specifying the moments, or angular accelerations, to be produced in pitch, roll, and yaw, rather than which particular control surface deflections to produce. How to transform these "virtual" control commands into physical control commands is known as the control allocation problem.

With a redundant actuator suite there are several combinations of actuator positions which all give (almost) the same overall system behavior. A common approach is to pick the combination that minimizes, e.g., control deflections, drag, wing load, or radar signature. ${ }^{3-7}$ In this paper we will use the redundancy to let different actuators produce control effort in different parts of the frequency spectrum. We will refer to this as dynamic control allocation.

Such frequency division may be desirable for at least two reasons. First, an actuator can be assigned a frequency range according to its intended operational use, e.g., for high, low, or midrange frequencies. Second, the high frequency control distribution, affecting the initial aircraft response to a pilot command, can be tuned without affecting the steady state control distribution, which may be designed to minimize, e.g., drag.

The remainder of this paper is organized as follows. In Section 2 the aircraft and actuator models to be used are introduced and motivated. Sec- tion 3 discusses the differences between static and dynamic allocation. The proposed control allocation method is presented in Section 4 and its properties, for the case when no actuator saturations occur, are analyzed in Section 5. A design example can be found in Section 6, and Section 7 contains some concluding remarks.

\section{$2 \quad$ Aircraft Model}

Let the aircraft dynamics be given by

$$
\begin{aligned}
\dot{x} & =f(x, \delta) \\
\dot{\delta} & =g(\delta, u)
\end{aligned}
$$

where $x=$ aircraft state vector, $\delta=$ actuator positions, and $u=$ actuator inputs. To incorporate the actuator position and rate constraints we impose that

$$
\begin{gathered}
\delta_{\min } \leq \delta \leq \delta_{\max } \\
|\dot{\delta}| \leq \delta_{\text {rate }}
\end{gathered}
$$

where $\delta_{\min }$ and $\delta_{\max }$ are the lower and upper position constraints, and $\delta_{\text {rate }}$ specifies the maximal individual actuator rates.

Even in the case when $f$ and $g$ are linear, it is nontrivial to design a control law which gives the desired closed loop dynamics while assuring that the actuator constraints are met. A common approach is to split the design task into two subtasks. To do this, we first use the fact that typically, control surface deflections primarily produce aerodynamic moment, $M(x, \delta)$. Second, the actuator 
dynamics are often very fast compared to the remaining aircraft dynamics, and can therefore be neglected. This gives us

$$
\begin{aligned}
& \delta \approx u \\
& \dot{x} \approx f_{M}(x, M(x, u))
\end{aligned}
$$

The control design can now be performed in two steps as follows. First, design a control law

$$
M(x, u)=k(r, x)
$$

where $r=$ pilot command, that yields some desired closed loop dynamics,

$$
\dot{x}=f_{M}(x, k(r, x))
$$

Second, determine $u$, constrained by (1) (with $\delta=$ $u$ ), that satisfies (2).

The latter step is the control allocation step. Since modern aircraft use digital flight control systems we rewrite (1b) in discrete time as

$$
|\dot{u}| \approx \frac{|u(t)-u(t-T)|}{T} \leq \delta_{\text {rate }}
$$

to get the overall position constraints at time $t$,

$$
\underline{u}(t) \leq u(t) \leq \bar{u}(t)
$$

where

$$
\begin{aligned}
& \underline{u}(t)=\max \left\{\delta_{\min }, u(t-T)-\delta_{\text {rate }} T\right\} \\
& \bar{u}(t)=\min \left\{\delta_{\max }, u(t-T)+\delta_{\text {rate }} T\right\}
\end{aligned}
$$

and $T$ is the sampling time. To simplify the search for a feasible solution we will assume the aerodynamic moment to be affine in the controls. This gives us

$$
M(x, u)=B(x) u+c(x)=k(r, x)
$$

or, equivalently,

$$
B u(t)=v(t)
$$

where

$$
v(t)=k(r, x)-c(x)
$$

is the virtual control input. Now, to perform online control allocation we need to find a feasible solution $u(t)$ at each sampling instant, satisfying (3) and (5). Figure 1 shows the structure of the resulting closed loop system.

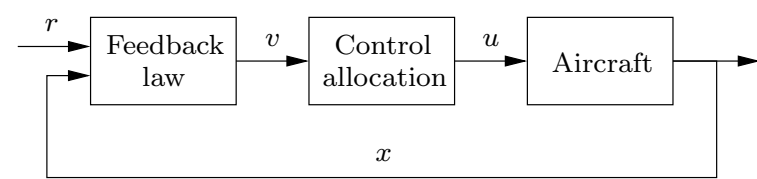

Fig. 1 Overview of the modular controller structure.

\section{Static vs Dynamic Control Allocation}

Several control allocation methods, like direct control allocation, ${ }^{8}$ daisy chaining, ${ }^{9}$ redistributed pseudoinverse ${ }^{3}$ and methods based on constrained quadratic $^{10,11}$ or linear ${ }^{7}$ programming, have been proposed in the literature, see ref. 12 for a survey. A common denominator for all these methods is that they are static in the sense that the physical control commands computed at time $t$, only depend on the virtual control commands at that time, i.e.,

$$
u(t)=f(v(t))
$$

Using a static mapping, no frequency division can be made between the actuators. To obtain a frequency division, and let different actuators produce control effort in different parts of the frequency spectrum, we need to use a dynamic mapping of the form

$$
\begin{aligned}
& u(t)=f(v(t), u(t-T), v(t-T), \\
&u(t-2 T), v(t-2 T), \ldots)
\end{aligned}
$$

With a dynamic mapping, the high frequency control distribution, affecting the initial aircraft response to a pilot command, and the low frequency control distribution, determining the distribution at steady state, need not be the same. Using static control allocation, on the other hand, a trade-off has to be made between good initial behavior and, e.g., low drag at trimmed flight.

In the following sections we will develop a strategy for performing dynamic control allocation using constrained quadratic programming. When no actuators saturate the relationship between $u$ and $v$ will be given by a first order linear filter of the form

$$
u(t)=F u(t-T)+G v(t)
$$

Previous efforts in this direction include ref. 13, where the required pitching moment is distributed to the tailerons through a low-pass filter and to the canard wings through a high-pass filter. This is motivated by the desire to get a fast initial response, produced by the canards, while the tailerons are known to produce more pitching moment, and are therefore used to generate the required moment at steady state. 
The difference between our approach and ref. 13 is twofold.

- To handle constraints on actuator positions and rates, we will perform the control allocation within a constrained quadratic programming framework. This ensures that (5) is satisfied whenever possible, since the control effort is redistributed when one actuator saturates.

- In a complex situation, where the number of moment generators is large, it is not an easy task to explicitly design the frequency distribution among the actuators while ensuring that (5) is satisfied. We propose the use of weighting matrices to affect the distribution of control effort, in size as well as in frequency, among the actuators.

\section{Dynamic Control Allocation Using QP}

The control allocation algorithm that we propose can be formulated as a linearly constrained quadratic programming problem:

$$
\begin{gathered}
\min _{u(t)}\left\|W_{1}\left(u(t)-u_{s}(t)\right)\right\|_{2}^{2}+ \\
\left\|W_{2}(u(t)-u(t-T))\right\|_{2}^{2} \\
B u(t)=v(t) \\
\underline{u}(t) \leq u(t) \leq \bar{u}(t)
\end{gathered}
$$

Equation (7a) is the cost function to be minimized under the linear constraints (7b) and (7c). Equation (7b) specifies which virtual control, $v$, to produce. We will assume $B$ to be an $n \times m$ matrix $(n<m)$ with rank $n$, where $n$ is the number of virtual controls (typically $n=3$ ) and $m$ is the number of physical controls available. Equation $(7 \mathrm{c})$ represents the feasible actuator positions at time $t$, regarding both the overall position constraints and the rate constraints as in (3).

Let us now focus on the cost function in (7a). $\|\cdot\|_{2}$ denotes the Euclidean 2-norm, i.e., $\|x\|_{2}=$ $\sqrt{x^{T} x}$ where $x$ is a column vector. $u_{s}(t)$ is the desired stationary distribution of control effort among the actuators and determines the actuator positions at trimmed flight. We will discuss the choice of $u_{s}$ in Section 5.3. $W_{1}$ and $W_{2}$ are weighting matrices whose $(i, i)$-entries specify whether it is important for the $i$ :th actuator, $u_{i}$, to quickly reach its desired stationary value, or to change its position as little as possible. With this interpretation, a natural choice is to use diagonal weighting matrices but in the analysis to follow we will allow arbitrary matrices with the following restriction.
Assumption 1 Assume that the weighting matrices $W_{1}$ and $W_{2}$ are symmetric and such that

$$
W=\sqrt{W_{1}^{2}+W_{2}^{2}}
$$

is nonsingular.

This assumption certifies that there is a unique optimal solution to the control allocation problem (7).

The difference between our approach and previous efforts based on quadratic programming is the second term in the cost function (7a), which penalizes the actuator rates. The two terms in the cost function can be merged into one term (see Lemma 2) without affecting the solution. Thus, any QP solver suitable for real-time implementation $^{3,10,11,14}$ can be used to find the solution.

How do the design variables, $u_{s}, W_{1}$, and $W_{2}$, affect the solution, $u(t)$ ?

\section{The Nonsaturated Case}

To answer this question, let us investigate the case where the optimal solution to $(7 \mathrm{a})-(7 \mathrm{~b})$ is feasible with respect to $(7 \mathrm{c})$. Then the actuator constraints can be disregarded and (7) reduces to

$$
\begin{gathered}
\min _{u(t)}\left\|W_{1}\left(u(t)-u_{s}(t)\right)\right\|_{2}^{2}+ \\
\left\|W_{2}(u(t)-u(t-T))\right\|_{2}^{2} \\
B u(t)=v(t)
\end{gathered}
$$

\subsection{Explicit solution}

Let us begin by stating the closed form solution to $(8)$.

Proposition 1 Let Assumption 1 hold. Then the control allocation problem (8) has the solution

$$
u(t)=E u_{s}(t)+F u(t-T)+G v(t)
$$

where

$$
\begin{aligned}
& E=(I-G B) W^{-2} W_{1}^{2} \\
& F=(I-G B) W^{-2} W_{2}^{2} \\
& G=W^{-1}\left(B W^{-1}\right)^{\dagger}
\end{aligned}
$$

The ${ }^{\dagger}$ symbol denotes the pseudoinverse operator defined as

$$
A^{\dagger}=A^{T}\left(A A^{T}\right)^{-1}
$$

for an $n \times m$ matrix $A$ with rank $n \leq m$.

The proposition shows that the optimal solution to the control allocation problem (8) is given by the first order linear filter (9). The properties of 
this filter will be further investigated in Sections 5.2 and 5.3.

In the remainder of this section we will develop a proof for Proposition 1.

Lemma 1 Let $A$ be an $n \times m$ matrix, where $n \leq$ $m$, with rank $n$. Then the minimum norm problem

$$
\begin{gathered}
\min _{x}\|x\|_{2} \\
A x=y
\end{gathered}
$$

has the solution

$$
x=A^{\dagger} y
$$

Proof: See, e.g., ref. 15.

Corollary 1 The weighted, shifted minimum norm problem

$$
\begin{gathered}
\min _{x}\left\|W\left(x-x_{0}\right)\right\|_{2} \\
A x=y
\end{gathered}
$$

where $W$ is nonsingular, has the solution

$$
\begin{aligned}
x & =F x_{0}+G y \\
F & =I-G A \\
G & =W^{-1}\left(A W^{-1}\right)^{\dagger}
\end{aligned}
$$

Proof: The change of variables

$$
e=W\left(x-x_{0}\right) \Longleftrightarrow x=x_{0}+W^{-1} e
$$

gives the equivalent problem

$$
\begin{gathered}
\min _{e}\|e\|_{2} \\
A\left(x_{0}+W^{-1} e\right)=y \Longleftrightarrow A W^{-1} e=y-A x_{0}
\end{gathered}
$$

Using Lemma 1, the solution is given by

$$
\begin{aligned}
e & =\left(A W^{-1}\right)^{\dagger}\left(y-A x_{0}\right) \Longleftrightarrow \\
x & =x_{0}+W^{-1}\left(A W^{-1}\right)^{\dagger}\left(y-A x_{0}\right) \\
& =\underbrace{\left(I-W^{-1}\left(A W^{-1}\right)^{\dagger} A\right)}_{F} x_{0}+\underbrace{W^{-1}\left(A W^{-1}\right)^{\dagger}}_{G} y
\end{aligned}
$$

Lemma 2 The cost function

$$
\left\|W_{1}\left(x-x_{1}\right)\right\|_{2}^{2}+\left\|W_{2}\left(x-x_{2}\right)\right\|_{2}^{2}
$$

has the same minimizing argument as

$$
\left\|W\left(x-x_{0}\right)\right\|_{2}
$$

where

$$
\begin{aligned}
& W=\sqrt{W_{1}^{2}+W_{2}^{2}} \\
& x_{0}=W^{-2}\left(W_{1}^{2} x_{1}+W_{2}^{2} x_{2}\right)
\end{aligned}
$$

\section{Proof:}

$$
\begin{aligned}
& \left\|W_{1}\left(x-x_{1}\right)\right\|_{2}^{2}+\left\|W_{2}\left(x-x_{2}\right)\right\|_{2}^{2}= \\
& \left(x-x_{1}\right)^{T} W_{1}^{2}\left(x-x_{1}\right)+\left(x-x_{2}\right)^{T} W_{2}^{2}\left(x-x_{2}\right)= \\
& x^{T}\left(W_{1}^{2}+W_{2}^{2}\right) x-2 x^{T}\left(W_{1}^{2} x_{1}+W_{2}^{2} x_{2}\right)+\ldots= \\
& \left(x-x_{0}\right)^{T} W^{2}\left(x-x_{0}\right)+\ldots=\left\|W\left(x-x_{0}\right)\right\|_{2}^{2}+\ldots
\end{aligned}
$$

where

$$
\begin{aligned}
& W=\sqrt{W_{1}^{2}+W_{2}^{2}} \\
& x_{0}=W^{-2}\left(W_{1}^{2} x_{1}+W_{2}^{2} x_{2}\right)
\end{aligned}
$$

The terms represented by dots do not depend on $x$, and hence they do not affect the minimization.

We are now ready to prove Proposition 1.

Proof: From Lemma 2 we know that the optimization criterion (8a) can be rewritten as

$$
\min _{u(t)}\left\|W\left(u(t)-u_{0}(t)\right)\right\|_{2}
$$

where

$$
\begin{aligned}
W & =\sqrt{W_{1}^{2}+W_{2}^{2}} \\
u_{0}(t) & =W^{-2}\left(W_{1}^{2} u_{s}(t)+W_{2}^{2} u(t-T)\right)
\end{aligned}
$$

Applying Corollary 1 to this criterion constrained by $(8 \mathrm{~b})$ yields

$$
\begin{aligned}
u(t) & =\bar{F} u_{0}(t)+G v(t) \\
\bar{F} & =I-G B \\
G & =W^{-1}\left(B W^{-1}\right)^{\dagger}
\end{aligned}
$$

from which it follows that

$$
\begin{aligned}
u(t)= & \underbrace{(I-G B) W^{-2} W_{1}^{2}}_{E} u_{s}(t)+ \\
& \underbrace{(I-G B) W^{-2} W_{2}^{2}}_{F} u(t-T)+G v(t)
\end{aligned}
$$

which completes the proof. 


\subsection{Dynamic properties}

Let us now study the dynamic properties of the filter (9). Note that the optimization criterion in (8) does not consider future values of $u(t)$. It is therefore not obvious that the resulting filter (9) should be even stable. The poles of the filter, which can be found as the eigenvalues of the feedback matrix $F$, are characterized by the following proposition.

Proposition 2 Let $F$ be defined as in Proposition 1 and let Assumption 1 hold. Then the eigenvalues of $F$ satisfy

$$
0 \leq \lambda(F) \leq 1
$$

If $W_{1}$ is nonsingular, the upper eigenvalue limit becomes strict, i.e.,

$$
0 \leq \lambda(F)<1
$$

Proof: We wish to characterize the eigenvalues of

$$
\begin{aligned}
F & =(I-G B) W^{-2} W_{2}^{2} \\
& =\left(I-W^{-1}\left(B W^{-1}\right)^{\dagger} B\right) W^{-2} W_{2}^{2} \\
& =W^{-1}\left(I-\left(B W^{-1}\right)^{\dagger} B W^{-1}\right) W^{-1} W_{2}^{2}
\end{aligned}
$$

Let the singular value decomposition of $B W^{-1}$ be given by

$$
B W^{-1}=U \Sigma V^{T}=U\left[\begin{array}{ll}
\Sigma_{r} & 0
\end{array}\right]\left[\begin{array}{l}
V_{r}^{T} \\
V_{0}^{T}
\end{array}\right]=U \Sigma_{r} V_{r}^{T}
$$

where $U$ and $V$ are unitary matrices, and $\Sigma_{r}$ is an $n \times n$ diagonal matrix with strictly positive diagonal entries (since $B W^{-1}$ has rank $n$ ). This yields

$$
\begin{gathered}
I-\left(B W^{-1}\right)^{\dagger} B W^{-1}=I-V_{r} \Sigma_{r}^{-1} U^{T} U \Sigma_{r} V_{r}^{T} \\
=I-V_{r} V_{r}^{T}=V_{0} V_{0}^{T}
\end{gathered}
$$

The last step follows from the fact that $V V^{T}=$ $V_{r} V_{r}^{T}+V_{0} V_{0}^{T}=I$. Inserting this into (11) gives us

$$
F=W^{-1} V_{0} V_{0}^{T} W^{-1} W_{2}^{2}
$$

Now use the fact ${ }^{16}$ that the nonzero eigenvalues of a matrix product $A B, \lambda_{n z}(A B)$, satisfy $\lambda_{n z}(A B)=\lambda_{n z}(B A)$ to get

$$
\lambda_{n z}(F)=\lambda_{n z}\left(V_{0}^{T} W^{-1} W_{2}^{2} W^{-1} V_{0}\right)
$$

From the definition of singular values we get

$$
\lambda\left(V_{0}^{T} W^{-1} W_{2}^{2} W^{-1} V_{0}\right)=\sigma^{2}\left(W_{2} W^{-1} V_{0}\right) \geq 0
$$

This shows that the nonzero eigenvalues of $F$ are real and positive and thus, $\lambda(F) \geq 0$ holds.

What remains to show is that the eigenvalues of $F$ are bounded by 1 . To do this we investigate the maximum eigenvalue, $\bar{\lambda}(F)$.

$$
\begin{aligned}
\bar{\lambda}(F) & =\bar{\sigma}^{2}\left(W_{2} W^{-1} V_{0}\right) \\
& =\left\|W_{2} W^{-1} V_{0}\right\|_{2}^{2} \\
& \leq\left\|W_{2} W^{-1}\right\|_{2}^{2}\left\|V_{0}\right\|_{2}^{2}
\end{aligned}
$$

Since

$$
\left\|V_{0}\right\|_{2}^{2}=\bar{\lambda}(\underbrace{V_{0}^{T} V_{0}}_{I})=1
$$

we get

$$
\begin{aligned}
\bar{\lambda}(F) & \leq\left\|W_{2} W^{-1}\right\|_{2}^{2} \\
& =\sup _{x \neq 0} \frac{x^{T} W^{-1} W_{2}^{2} W^{-1} x}{x^{T} x}= \\
& =\sup _{y \neq 0} \frac{y^{T} W_{2}^{2} y}{y^{T} W^{2} y} \\
& =\sup _{y \neq 0} \frac{y^{T} W_{2}^{2} y}{y^{T} W_{1}^{2} y+y^{T} W_{2}^{2} y} \\
& \leq \sup _{y \neq 0} \frac{y^{T} W_{2}^{2} y}{y^{T} W_{2}^{2} y}=1
\end{aligned}
$$

since $y^{T} W_{1}^{2} y=\left\|W_{1} y\right\|_{2}^{2} \geq 0$ for any symmetric $W_{1}$. If $W_{1}$ is nonsingular, we get $y^{T} W_{1}^{2} y=$ $\left\|W_{1} y\right\|_{2}^{2}>0$ for $y \neq 0$ and the last inequality in (12) becomes strict, i.e., $\bar{\lambda}(F)<1$ in this case.

The proposition states that the poles of the linear control allocation filter (9) lie between 0 and 1 on the real axis. This has two important practical implications:

- If $W_{1}$ is nonsingular the filter poles lie strictly inside the unit circle. This implies that the filter is asymptotically stable. $W_{1}$ being nonsingular means that all actuator positions except $u_{s}$ render a nonzero cost in (7a). If $W_{1}$ is singular, only marginal stability can be guaranteed (although asymptotic stability may hold).

- The fact that the poles lie on the positive real axis implies that the actuator responses to a step in the virtual control input are not oscillatory.

\subsection{Steady state properties}

In the previous section we showed that the control allocation filter (9) is asymptotically stable 
under practically reasonable assumptions. Let us therefore investigate the steady state solution to a step response.

Proposition 3 Let $u_{s}$ be a constant feasible solution such that

$$
B u_{s}=v_{0}
$$

where $v(t) \equiv v_{0}$ is the desired virtual control input. Then, if (9) is an asymptotically stable filter, the steady state control distribution is given by

$$
\lim _{t \rightarrow \infty} u(t)=u_{s}
$$

Proof: If the linear filter (9) is asymptotically stable we know that the limit value

$$
u_{\infty}=\lim _{t \rightarrow \infty} u(t)
$$

exists. Setting $u(t)=u(t-T)=u_{\infty}$ in (9) and using $B u_{s}=v_{0}$ yields

$$
u_{\infty}=(I-F)^{-1}(E+G B) u_{s}
$$

Using the identity $W^{2}=W_{1}^{2}+W_{2}^{2}$ we get

$$
\begin{aligned}
E+G B & =(I-G B) W^{-2} W_{1}^{2}+G B \\
& =(I-G B)\left(I-W^{-2} W_{2}^{2}\right)+G B \\
& =I-(I-G B) W^{-2} W_{2}^{2}=I-F
\end{aligned}
$$

which gives the desired result

$$
u_{\infty}=(I-F)^{-1}(I-F) u_{s}=u_{s}
$$

Thus, if we feed our dynamic control allocation scheme with a feasible, desirable control distribution, $u_{s}$, which solves $B u_{s}=v$, the filter (9) will render this distribution at steady state.

If $u_{s}$ is not a feasible solution, the resulting steady state control distribution will depend not only on $u_{s}$, but also on $W_{1}$ as well as $W_{2}$. This is undesirable since the role of the different design parameters then becomes unclear.

So how do we find a good feasible steady state solution? In simple cases, we may be able to do it by hand but for larger cases the following method can be applied. Pick $u_{s}$ as the solution to the static control allocation problem

$$
\begin{gathered}
\min _{u_{s}}\left\|W_{s}\left(u_{s}-u_{p}\right)\right\|_{2} \\
B u_{s}=v
\end{gathered}
$$

Here, $u_{p}$ represents some fixed preferred, but typically infeasible control distribution, which, e.g.,

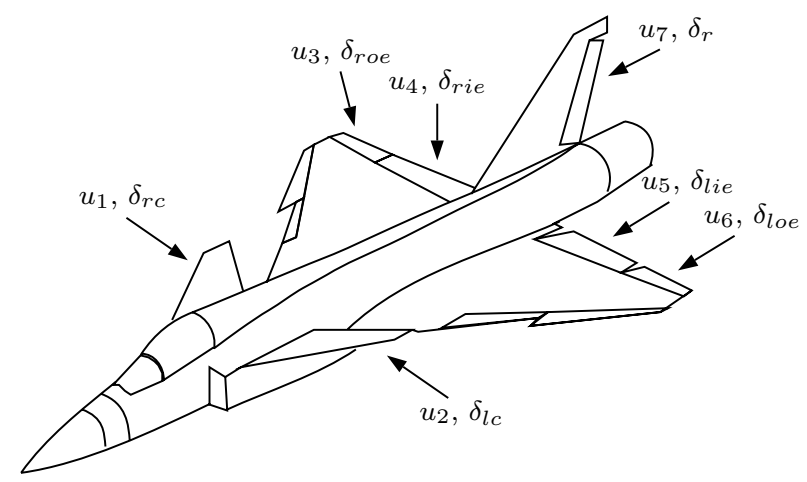

Fig. 3 Admire control surface configuration.

would give minimum drag. In the simplest case, with $W_{s}=I$ and $u_{p}=0$, we get the pseudoinverse solution $u_{s}=B^{\dagger} v$.

In certain cases, some steady state actuator positions should be scheduled with, e.g., speed and altitude, rather than depend on $v$. This can be handled by introducing additional equality constraints

$$
u_{s, i}=u_{p, i}
$$

for those actuators $i$ whose steady state positions have been predetermined. The optimal solution to (13)-(14) can be found using Corollary 1.

\section{Design Example}

Let us now illustrate how to use the proposed design method for dynamic control allocation. The Admire model, ${ }^{17}$ a Simulink based realistic fighter aircraft model including, e.g., actuator dynamics and nonlinear aerodynamics, is used for simulation. The existing flight control system is used to compute the aerodynamic moment coefficients, $k(r, x)=M\left(x, u_{\mathrm{Adm}}\right)$, to be produced in roll, pitch, and yaw, see Figure 2 . The model parameters $B$ and $c$ in (4), used in (5) and (6), are computed at each sampling instant by linearizing $M(x, u)$ around the current measurement vector, $x(t)$, and the previous control vector, $u(t-T)$. In the Admire model, $T=0.02 \mathrm{~s}$. The constrained QP problem (7) is solved at each sampling instant using the sequential least squares solver from ref. 14 .

The control vector,

$$
u=\left(\begin{array}{lll}
u_{1} & \ldots & u_{7}
\end{array}\right)^{T}
$$

consists of the commanded deflections for the canard wings (left and right), the elevons (inboard and outboard, left and right), and for the rudder, in radians, see Figure 3 where $\delta_{*}$ denote the actual actuator positions. The actuator constraints 


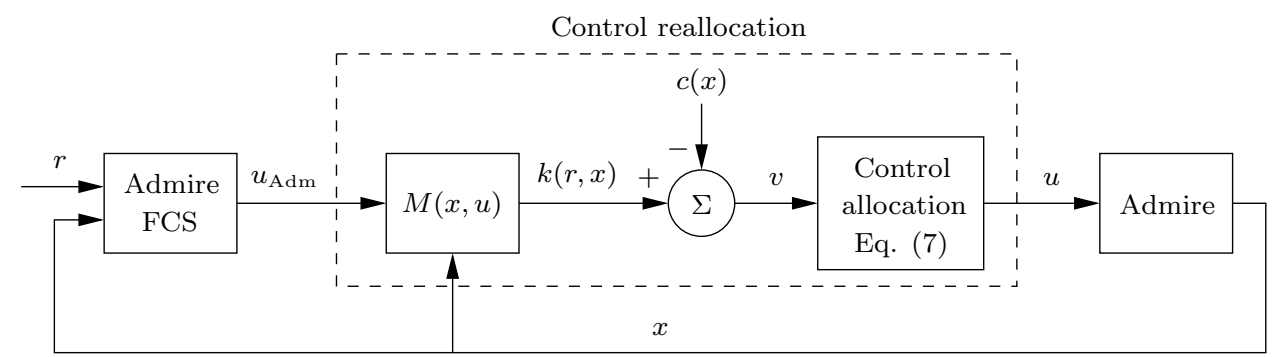

Fig. 2 Overview of the closed loop system used for simulation. The controls produced by the Admire flight control system are reallocated using dynamic control allocation.

are given by

$$
\begin{aligned}
\delta_{\min } & =\left(\begin{array}{llllllll}
-55 & -55 & -30 & -30 & -30 & -30 & -30
\end{array}\right)^{T} \\
\delta_{\max } & =\left(\begin{array}{llllllll}
25 & 25 & 30 & 30 & 30 & 30 & 30
\end{array}\right)^{T} \\
\delta_{\text {rate }} & =\left(\begin{array}{lllllll}
50 & 50 & 150 & 150 & 150 & 150 & 100
\end{array}\right)^{T}
\end{aligned}
$$

measured in degrees, and degrees per second, respectively.

At trimmed flight at Mach $0.5,1000 \mathrm{~m}$, the control effectiveness matrix is given by

$$
\begin{aligned}
& B=10^{-2} \times \\
& \left(\begin{array}{ccccccc}
0.5 & -0.5 & -4.9 & -4.3 & 4.3 & 4.9 & 2.4 \\
8.8 & 8.8 & -8.4 & -13.8 & -13.8 & -8.4 & 0 \\
-1.7 & 1.7 & -0.5 & -2.2 & 2.2 & 0.5 & -8.8
\end{array}\right)
\end{aligned}
$$

from which it can be seen, e.g., that the inboard elevons are the most effective actuators for producing pitching moment while the rudder provides good yaw control, as expected.

Let us now consider the requirements regarding the control distribution. At trimmed flight, it is beneficial not to deflect the canards at all to achieve minimum drag. We therefore select the steady state distribution $u_{s}$ as the solution to

$$
\begin{gathered}
\min _{u_{s}}\left\|u_{s}\right\|_{2} \\
B u_{s}=v \\
u_{s, 1}=u_{s, 2}=0
\end{gathered}
$$

which yields

$$
u_{s}=S v
$$

where

$$
S=\left(\begin{array}{ccc}
0 & 0 & 0 \\
0 & 0 & 0 \\
-5.4 & -1.6 & -0.4 \\
-4.6 & -2.6 & -2.4 \\
4.6 & -2.6 & 2.4 \\
5.4 & -1.6 & 0.4 \\
3.0 & 0 & -10.1
\end{array}\right)
$$

During the initial phase of a pitch maneuver, on the other hand, utilizing the canards counteracts the unwanted nonminimum phase tendencies that the pilot load factor, $n_{z}$, typically displays. Thus, the canards should be used to realize parts of the high frequency content of the pitching moment. Selecting

$$
\begin{aligned}
& W_{1}=\operatorname{diag}\left(\begin{array}{lllllll}
2 & 2 & 2 & 2 & 2 & 2 & 2
\end{array}\right) \\
& W_{2}=\operatorname{diag}\left(\begin{array}{lllllll}
5 & 5 & 10 & 10 & 10 & 10 & 10
\end{array}\right)
\end{aligned}
$$

with the lowest rate penalty on the canards, and using Proposition 1, yields the control allocation filter

$$
u(t)=F u(t-T)+G_{t o t} v(t)
$$

where

$$
\begin{aligned}
F=10^{-1} \times & \\
& \left(\begin{array}{ccccccc}
5.5 & -1.4 & 2.9 & 3.4 & 4.3 & 1.8 & -5.0 \\
-1.4 & 5.5 & 1.8 & 4.3 & 3.4 & 2.9 & 5.0 \\
0.7 & 0.4 & 6.4 & -3.4 & 1.3 & 1.9 & 0.7 \\
0.9 & 1.1 & -3.4 & 5.5 & 0.7 & 1.3 & -0.8 \\
1.1 & 0.9 & 1.3 & 0.7 & 5.5 & -3.4 & 0.8 \\
0.4 & 0.7 & 1.9 & 1.3 & -3.4 & 6.4 & -0.7 \\
-1.3 & 1.3 & 0.7 & -0.8 & 0.8 & -0.7 & 2.2
\end{array}\right) \\
& \\
G_{\text {tot }}=G+E S & =\left(\begin{array}{cccc}
1.7 & 2.8 & -5.3 \\
-1.7 & 2.8 & 5.4 \\
-5.3 & -0.8 & -0.6 \\
-4.7 & -1.3 & -2.2 \\
4.7 & -1.3 & 2.2 \\
5.3 & -0.8 & 0.6 \\
2.4 & 0 & -8.2
\end{array}\right)
\end{aligned}
$$

in the nonsaturated case. Figure 5 shows a magnitude plot of the transfer functions from $v$ to $u$. Each transfer function has been weighted with its corresponding entry in $B$ to show the proportion of $v$ that the actuator produces. In roll, the elevons produce most of the control needed while in pitch, the canards contribute substantially at high frequencies. Yaw control is produced almost exclusively by the rudder.

Figure 6 shows the simulation results from a pitch up command followed by a roll command. It is worth pointing out that the discrepancies between $p$ and $p_{\text {com }}$, and between $q$ and $q_{\text {com }}$, are not due to the choice of control allocation algorithm, 


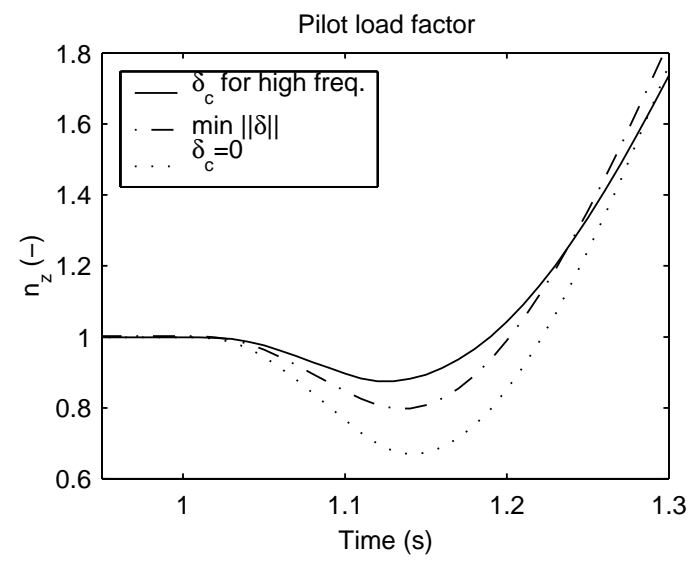

Fig. 4 Comparison of the nonminimum phase behavior in $n_{z}$ for different control allocation strategies when a pitch command is applied. Using the canards to produce high frequency pitching moment yields a small undershoot in $n_{z}$ while minimizing the drag at trimmed flight.

but arise from the design of the Admire control system.

In accordance with the designed frequency distributions, the canard wings react quickly to the pitch command while at steady state only the elevons and the rudder are deflected.

The gain from using the canards in this fashion can be seen in Figure 4 which shows a blowup of the load factor behavior at $t=1 \mathrm{~s}$ when different control allocation strategies are used. The dotted curve, with the largest undershoot, arises when the canards are not used at all. The dashdotted curve represents static control allocation with a minimal control objective $\left(u_{s}=0, W_{1}=I\right.$, and $W_{2}=0$ in (7)). The undershoot is decreased but the drag at trimmed flight is increased by $2 \%$ since the canard deflections are nonzero at steady state. The solid curve coincides with that in Figure 6 and results from using dynamic control allocation. This yields the smallest undershoot with no increase of drag at trimmed flight since at steady state the canard delections are zero.

\section{Conclusions}

The proposed method for dynamic control allocation offers the user significant freedom in designing the control distribution among the actuators not only in size but also in frequency. A main advantage compared to static allocation methods is that the high frequency control distribution, affecting the initial aircraft response to a pilot command, and the steady state control distribution, determining, e.g., drag and radar signature
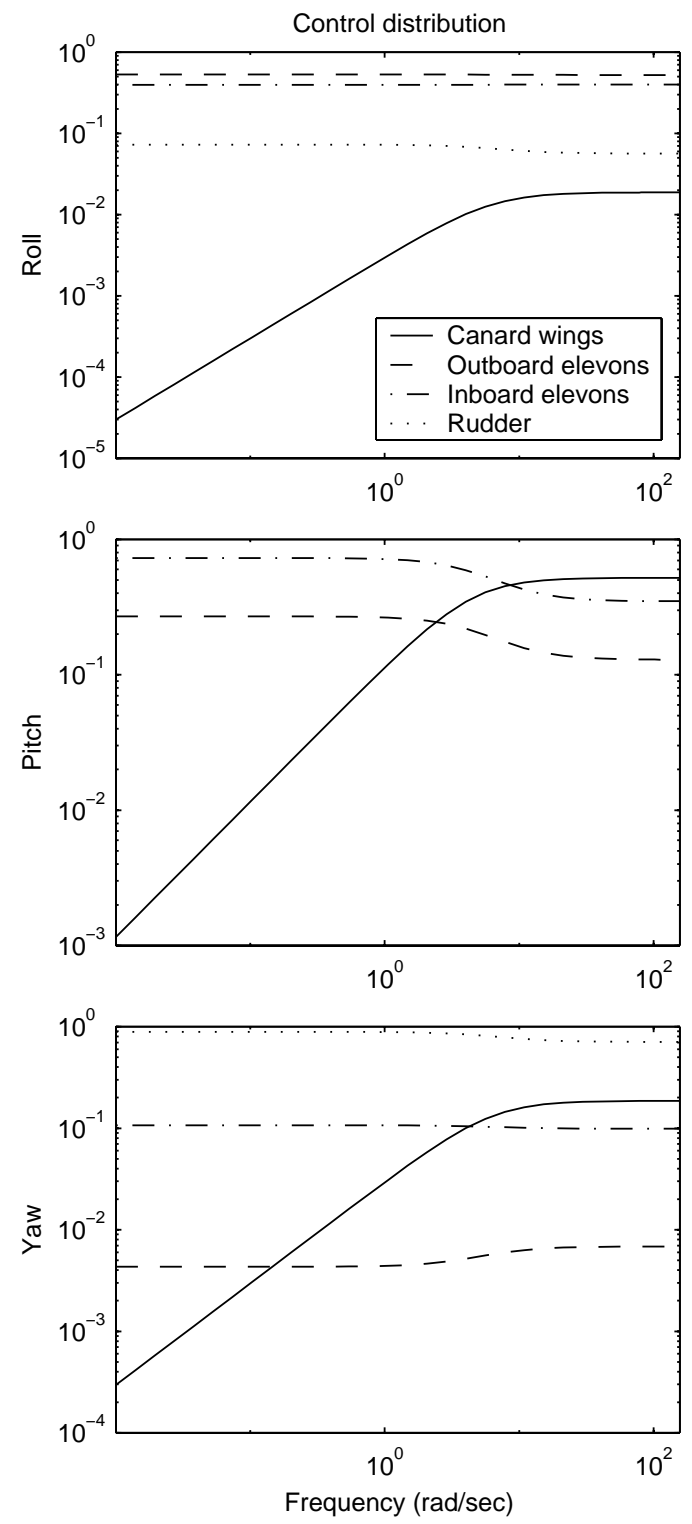

Fig. 5 Distribution of control effort among the actuators at different frequencies. The curves represent the magnitudes of the transfer functions from $v$ to $u$. Each transfer function has been weighted with its corresponding entry in $B$ to show the proportion of $v$ that the actuator produces.

at trimmed flight can be selected different. Performing the filtering in a constrained optimization framework provides automatic redistribution of control effort when one actuator saturates in position or in rate. 

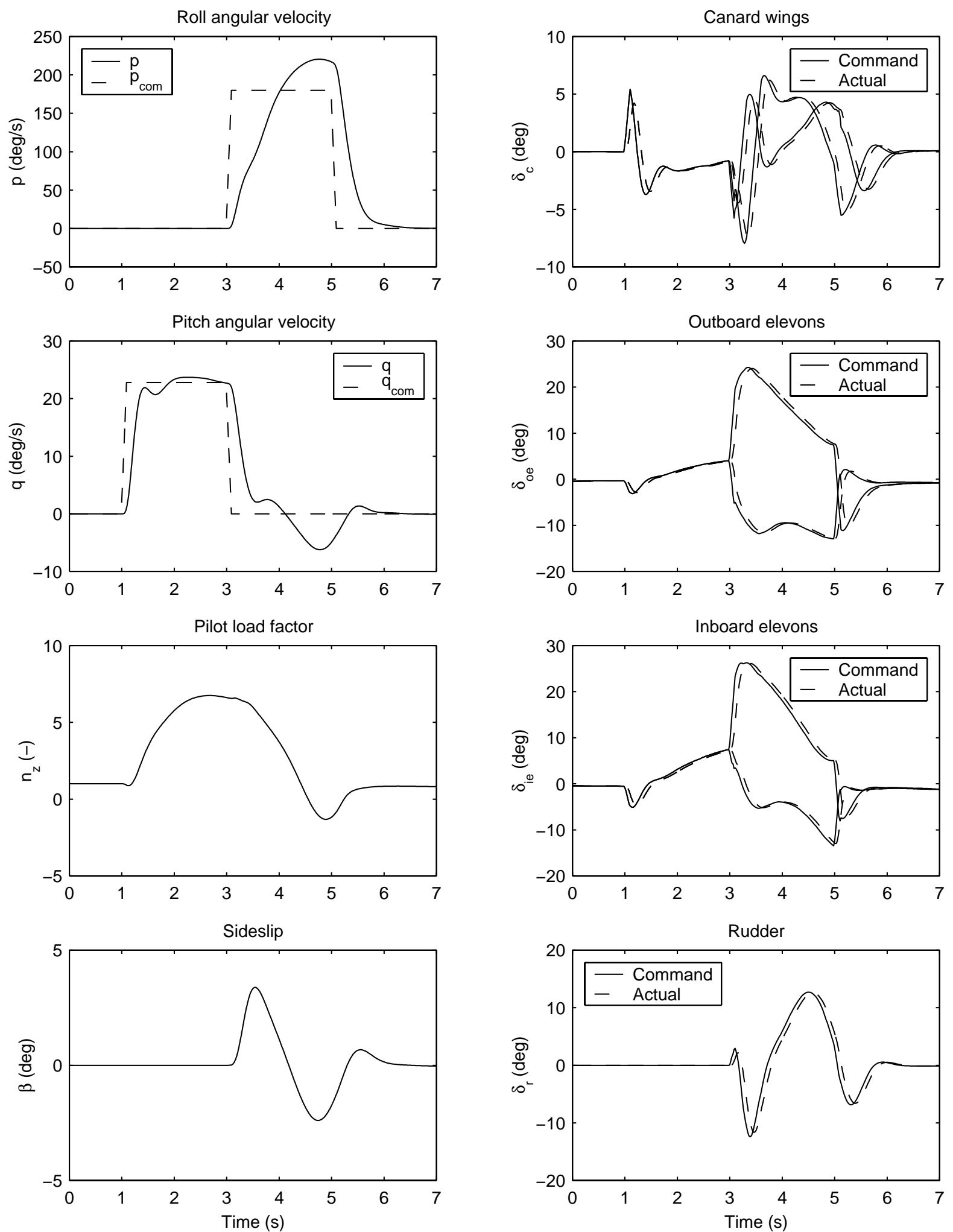

Fig. 6 Aircraft trajectory and control surface deflections. For the control surface deflections, both the commanded deflections and the actual deflections, considering actuator position and rate constraints and also actuator dynamics, are shown. 


\section{References}

${ }^{1}$ Enns, D., Bugajski, D., Hendrick, R., and Stein, G., "Dynamic inversion: an evolving methodology for flight control design," International Journal of Control, Vol. 59, No. 1, Jan. 1994, pp. 71-91.

${ }^{2}$ Härkegård, O. and Glad, S. T., "Flight control design using backstepping," Proc. of the IFAC NOLCOS'01, St. Petersburg, Russia, July 2001.

${ }^{3}$ Virnig, J. C. and Bodden, D. S., "Multivariable control allocation and control law conditioning when control effectors limit," AIAA Guidance, Navigation, and Control Conference and Exhibit, Scottsdale, AZ, Aug. 1994.

${ }^{4}$ Buffington, J. M., "Tailless aircraft control allocation," AIAA Guidance, Navigation, and Control Conference and Exhibit, New Orleans, LA, 1997, pp. 737-747.

${ }^{5}$ Wise, K. A., Brinker, J. S., Calise, A. J., Enns, D. F., Elgersma, M. R., and Voulgaris, P., "Direct adaptive reconfigurable flight control for a tailless advanced fighter aircraft," International Journal of Robust and Nonlinear Control, Vol. 9, No. 14, 1999, pp. 999-1012.

${ }^{6}$ Eberhardt, R. L. and Ward, D. G., "Indirect adaptive flight control of a tailless fighter aircraft," AIAA Guidance, Navigation, and Control Conference and Exhibit, Portland, OR, 1999, pp. 466-476.

${ }^{7}$ Ikeda, Y. and Hood, M., "An application of L1 optimization to control allocation," AIAA Guidance, Navigation, and Control Conference and Exhibit, Denver, CO, Aug. 2000.

${ }^{8}$ Durham, W. C., "Constrained control allocation: Three moment problem," Journal of Guidance, Control, and Dynamics, Vol. 17, No. 2, March-April 1994, pp. 330336.

${ }^{9}$ Buffington, J. M. and Enns, D. F., "Lyapunov stability analysis of daisy chain control allocation," Journal of Guidance, Control, and Dynamics, Vol. 19, No. 6, Nov.Dec. 1996, pp. 1226-1230.

${ }^{10}$ Burken, J. J., Lu, P., Wu, Z., and Bahm, C., "Two reconfigurable flight-control design methods: Robust servomechanism and control allocation," Journal of Guidance, Control, and Dynamics, Vol. 24, No. 3, May-June 2001, pp. 482-493.

${ }^{11}$ Enns, D., "Control allocation approaches," $A I A A$ Guidance, Navigation, and Control Conference and Exhibit, Boston, MA, 1998, pp. 98-108.

${ }^{12}$ Bodson, M., "Evaluation of optimization methods for control allocation," AIAA Guidance, Navigation, and Control Conference and Exhibit, Montreal, Canada, Aug. 2001.

${ }^{13}$ Papageorgiou, G., Glover, K., and Hyde, R. A., "The $\mathcal{H}_{\infty}$ loop-shaping approach," Robust Flight Control: A Design Challenge, edited by J.-F. Magni, S. Bennani, and J. Terlouw, chap. 29, Springer, 1997, pp. 464-483.

${ }^{14}$ Härkegård, O., "Efficient active set algorithms for solving constrained least squares problems in aircraft control allocation," Tech. Rep. LiTH-ISY-R-2426, Department of Electrical Engineering, Linköpings universitet, SE-581 83 Linköping, Sweden, May 2002, Available at http://www. control.isy.liu.se.

${ }^{15}$ Golub, G. H. and Loan, C. F. V., Matrix Computations, John Hopkins University Press, 2nd ed., 1989.

${ }^{16}$ Zhang, F., Matrix Theory: Basic results and techniques, Springer, 1999.

${ }^{17}$ Swedish Defence Research Agency (FOI), "Aerodata Model in Research Environment (Admire)," http://www.foi.se/admire/.

American Institute of Aeronautics and Astronautics 